\title{
Nanostructural Effect of ZnO on Light Extraction Efficiency of Near-Ultraviolet Light-Emitting Diodes
}

\author{
Young Jae Park, ${ }^{1}$ Hyounsuk Song, ${ }^{1}$ Kang Bok Ko, ${ }^{1}$ Beo Deul Ryu, \\ Tran Viet Cuong, ${ }^{1,2}$ and Chang-Hee Hong ${ }^{1}$ \\ ${ }^{1}$ School of Semiconductor and Chemical Engineering, Semiconductor Physics Research Center, Chonbuk National University, \\ 20 Geonji-ro, Deokjin-gu, Jeonju-si 54907, Republic of Korea \\ ${ }^{2}$ NTT Hi-Tech Institute, Nguyen Tat Thanh University, 298-300 A Nguyen Tat Thanh Street, Ho Chi Minh City, Vietnam
}

Correspondence should be addressed to Tran Viet Cuong; tvcuong@ntt.edu.vn and Chang-Hee Hong; chhong@jbnu.ac.kr

Received 1 December 2015; Accepted 17 February 2016

Academic Editor: Takuya Tsuzuki

Copyright (C) 2016 Young Jae Park et al. This is an open access article distributed under the Creative Commons Attribution License, which permits unrestricted use, distribution, and reproduction in any medium, provided the original work is properly cited.

\begin{abstract}
The effect of $\mathrm{ZnO}$ nanostructures on the light output power of $375 \mathrm{~nm}$ near-ultraviolet light-emitting diodes (NUV-LEDs) was investigated by comparing one-dimensional (1D) nanorods (NR-ZnO) with two-dimensional (2D) nanosheets (NS- $\mathrm{ZnO})$. ZnO nanostructures were grown on a planar indium tin oxide (ITO) by solution based method at low temperature of $90^{\circ} \mathrm{C}$ without degradation of the forward voltage. At an injection current of $100 \mathrm{~mA}$, the light output efficiency of NUV-LED with NR-ZnO was enhanced by around 30\% compared to the conventional NUV-LEDs without $\mathrm{ZnO}$ nanostructures. This improvement is due to the formation of a surface texturing, resulting in a larger escape cone and a multiple scattering for the photons in the NUV-LED, whereas the light output efficiency of NUV-LED with NS-ZnO was lower than that of the conventional NUV-LEDs due to the internal reflection and light absorption in the defective sites of NS- $\mathrm{ZnO}$.
\end{abstract}

\section{Introduction}

An attempt to improve the performance of AlGaN based near-ultraviolet light-emitting diodes (NUV-LEDs) has attracted much attention due to their great potential for applications such as water purification, biochemical detection, and being a source for white Hg-free lamps [1-3]. However, UV-LEDs have suffered from low external quantum efficiency due to high electrical activation energy for $\mathrm{Mg}$ doped $p-\mathrm{GaN}$, low optical transmittance of transparent conductive layers, and low internal quantum efficiency. Several studies have reported improving the crystal quality of AlGaN by using AlN interlayer $[4,5]$ and AlN/AlGaN superlattices to enhance the optoelectrical performance of the NUV-LED, and using modified graphene [6, 7]. However, these researches only focused on the improvement of the crystal quality and have a large turn-on voltage with inefficient current spreading. An increase in the light extraction efficiency of NUV-LED without degradation of electrical properties is rarely discussed. One of the challenges which need to be overcome is the low light extraction efficiency, owing to the high internal reflection and narrow escape cone $\left(\sim 23.6^{\circ}\right)$ due to the large difference of refractive indexes between nitride material $\left(\eta_{\mathrm{GaN}}=2.5\right)$ and air $\left(\mu_{\text {air }}=1\right)$. In the past decade, several methods have been proposed for releasing the photons trapped inside the LEDs, including flip-chip LEDs [8], photonic crystals [9], and surface roughening $[10,11]$. However, these methods involve complex, costly, long fabrication and dry etching process which may result in degradation of the electrical properties due to surface damage [12]. Alternatively, our previous study revealed that light extraction can be enhanced without degradation of electrical properties by using an aqueous solution method to grow $\mathrm{ZnO}$ nanostructures on top of LEDs. The use of $\mathrm{ZnO}$ nanostructures not only significantly increases the escape cones since the well-matched refractive indexes between $\mathrm{ZnO}$ and $\mathrm{GaN}$ materials but also allows emitted photons to escape through sidewall and rough surface due to surface scattering at the interface. Although aqueous solution is considered a facile, scalable, low 


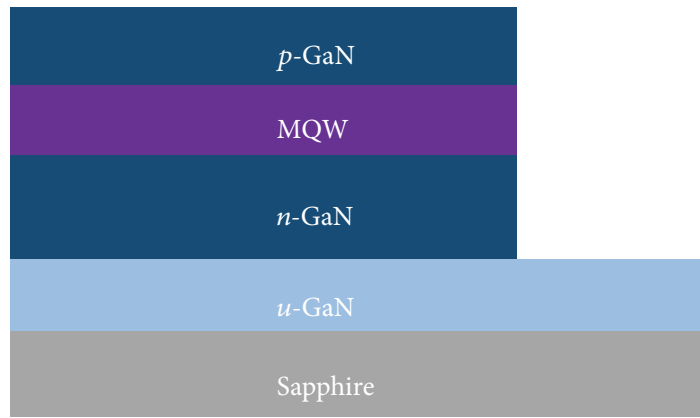

(a)

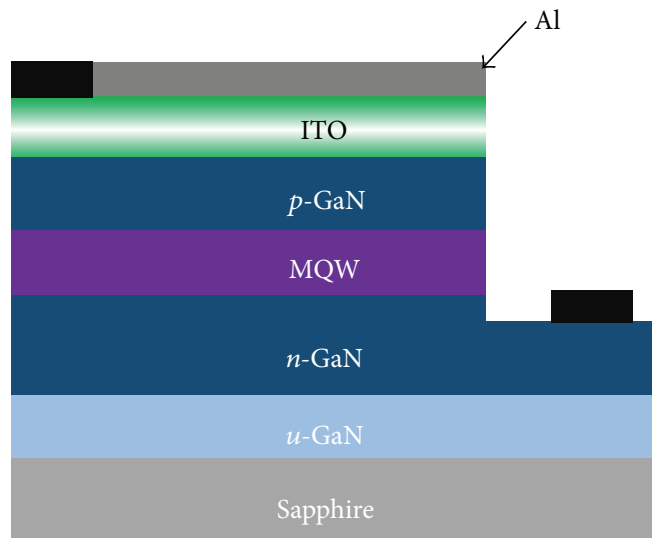

(c)

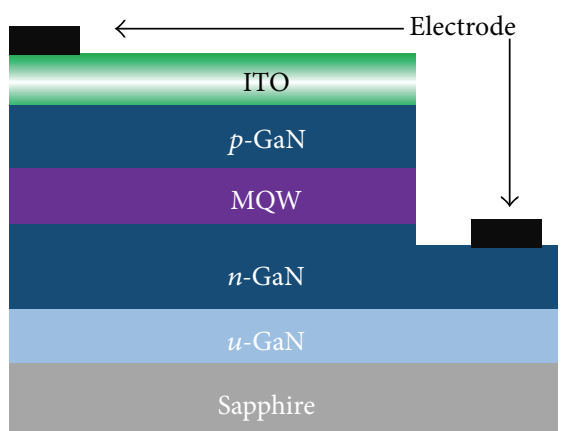

(b)

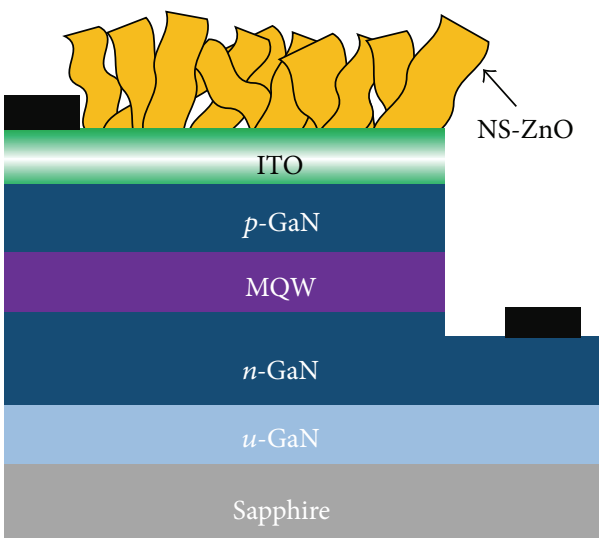

(d)

FIGURE 1: Schematic diagram of the process flow: (a) mesa etching after the UVLED structure growth on sapphire substrate, (b) deposition of bonding metal electrode after deposition of ITO layer onto $p$-GaN, (c) selective deposition of thin Al layer on the ITO area using a photolithography, and (d) $\mathrm{ZnO}$ nanostructure growth by a hydrothermal method.

temperature, and cost effective method, difficulties in controlling crystalline structure and morphology are the main obstacles to realizing this approach. It is believed that elucidating the effect of nanostructure morphology on light extraction efficiency of LED devices is very important for the development of NUV-LEDs with high luminance efficiency. In this work, two different nanostructures of $\mathrm{ZnO}$, nanorods (NR) and nanosheets (NS), have been employed to investigate their effect on light extraction efficiency of NUV-LED devices.

\section{Experimental}

Figure 1 shows the schematic diagram of the processes for fabricating the UVLED with $\mathrm{ZnO}$ nanostructures. $\mathrm{ZnO}$ nanostructures were formed onto ITO films deposited on NUVLED structures. The NUV-LED structure was grown on a 2 in. diameter $c$-plane sapphire substrate using metal organic chemical vapor deposition. Trimethylgallium (TMGa) and trimethylaluminum (TMAl) were used as $\mathrm{Ga}$ and $\mathrm{Al}$ metal organic precursors, and ammonia $\left(\mathrm{NH}_{3}\right)$ was used as a nitrogen source. Bis(cyclopentadienyl) magnesium $\left(\mathrm{Cp}_{2} \mathrm{Mg}\right)$ and silane $\left(\mathrm{SiH}_{4}\right)$ were employed for $p$-type and $n$-type dopant sources, respectively. The device structure is $p$ $\mathrm{GaN}(0.15 \mu \mathrm{m}) / \mathrm{AlGaN}$ electron blocking layer/a 5-period InGaN/AlGaN multiple quantum wells (MQWs)/n-GaN
$(2 \mu \mathrm{m}) /$ undoped GaN/sapphire. After mesa etching, a $100 \mathrm{~nm}$ ITO layer was deposited onto $p$-GaN by e-beam evaporation and was annealed at $500^{\circ} \mathrm{C}$ for $30 \mathrm{sec}$ under $\mathrm{N}_{2}: \mathrm{O}_{2}$ $(8: 2)$ mixed ambient. The bonding pad electrodes of $\mathrm{Cr} / \mathrm{Au}$ $(30 / 250 \mathrm{~nm})$ were deposited onto the top surface of the ITO transparent electrode and $n$-type $\mathrm{GaN}$ by e-beam evaporation. We fabricated InGaN/AlGaN MQW NUV-LEDs with conventional planar ITO (C-LED), ZnO nanorod array/ITO (NR-LED), and ZnO nanosheet array/ITO (NS-LED). To realize $\mathrm{ZnO}$ nanomaterials on an ITO electrode, the ITO area of the p-electrode was made open by a photoresist lift-off process. NR- and NS-ZnO were formed by a hydrothermal growth method. This method creates the nanostructures by using an aqueous solution at $90^{\circ} \mathrm{C}$ on the transparent contact layer of ITO. The solution to grow the $\mathrm{ZnO}$ nanorods (NR$\mathrm{ZnO}$ ) and $\mathrm{ZnO}$ nanosheets (NS- $\mathrm{ZnO}$ ) is described in detail elsewhere [13].

The morphologies and structures of the $\mathrm{ZnO}$ nanomaterials arrays were characterized via scanning electron microscopy (SEM, S-4700, Hitachi, Japan). The optical properties were investigated using photoluminescence spectra with a He-Cd excited source $(325 \mathrm{~nm})$, and light output power measured as a function of applied current $(L-I)$ was carried out using an optical detector connected to a parameter analyzer. The current-voltage $(I-V)$ characteristics were 


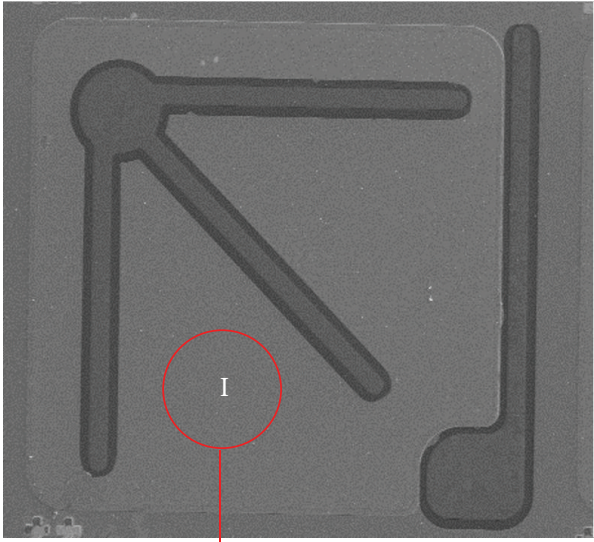

(a)

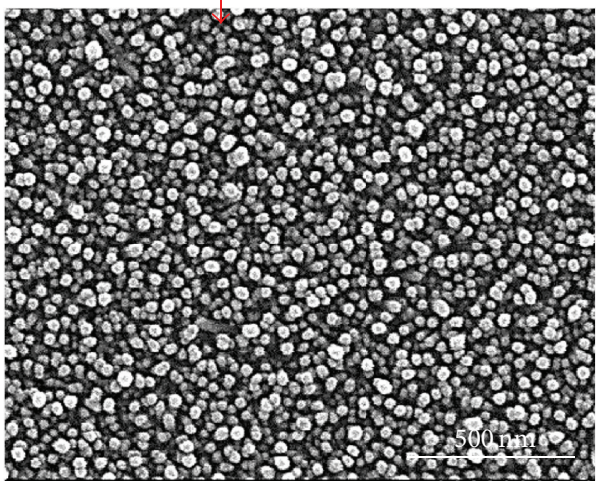

(c)

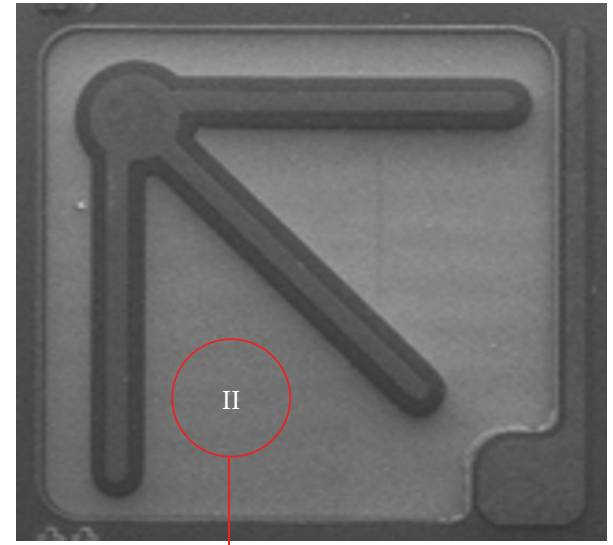

(b)

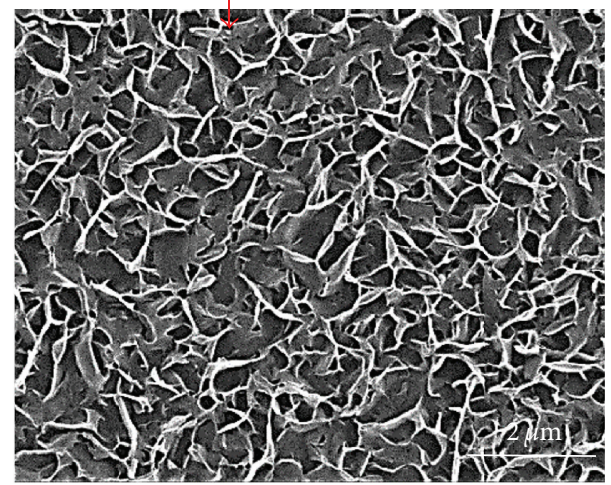

(d)

FIgURE 2: Top-view SEM images of ((a)-(b)) electrode pad configuration of NR-LED and NS-LED with two defined areas (c) and (d), respectively.

measured using a semiconductor characterization system (Keithley 4200-MSTech).

\section{Results and Discussion}

Figure 2 indicates that the $\mathrm{ZnO}$ nanostructures are well defined and grown on top of NUV-LED structures via hydrothermal growth method. In order to realize NR-ZnO on the ITO layer selectively, a conventional photolithography technique was performed to make the ITO layer open except the electrodes. A synthesized Ga-doped ZnO (GZO) solution [13] was spin-coated onto the substrate at $3000 \mathrm{rpm}$ for $20 \mathrm{~s}$ to form a GZO buffer layer. The GZO buffer layer coated substrate was then annealed at $350^{\circ} \mathrm{C}$ in air for $60 \mathrm{~min}$ to form a seed layer for NR-ZnO. To grow NS-ZnO, a $10 \mathrm{~nm}$ thick $\mathrm{Al}$ was deposited onto ITO layer using e-beam evaporation. The NR-ZnO on GZO seeds and the NS-ZnO on Al thin film were grown by a dipping and holding process into a mixed solution containing DI water, $18 \mathrm{mM}$ zinc nitrate hexahydrate, and $20 \mathrm{mM}$ hexamethylenetetramine for $90 \mathrm{~min}$ at $90^{\circ} \mathrm{C}$.

Figures 2(a) and 2(b) show a plan-view optical micrograph of the NR- and NS-LED, respectively. The $\mathrm{ZnO}$ nanostructures were selectively grown on ITO surface area as shown in regions I and II. In addition, clear pattern of $\mathrm{Cr} / \mathrm{Au}$ electrode pad as well as ITO transparent conductive layer was also observed. The distinct surface morphologies of NR and NS are clearly exhibited in Figures 2(c) and 2(d). Figure 2(c) shows the typical SEM image of single crystalline NR-ZnO with orientation preferably vertical to the ITO surface. The length and the diameter of the nanorods are in the range of 40-50 and 200-250 nm, respectively. Figure 2(d) shows the NS-ZnO structure on ITO layer. The nanosheets have a length of about $500 \mathrm{~nm}$. The two-dimensional growth of NS$\mathrm{ZnO}$ is formed as a result of the $\mathrm{Al}$ element. The $\mathrm{Al}$ film deposited onto ITO layer had been incorporated into $\mathrm{ZnO}$ during hydrothermal reaction process and suppressed the growth along [001] direction. The primary reason is that the $\mathrm{Al}$ atom inhibited the growth rate of the polar surface by adsorbing; therefore, the growth rate of the nonpolar surface is faster than that of the polar surface [14].

Figure 3(a) shows UV-Vis spectra of the ITO, NS-ZnO, and NR-ZnO. The optical transmittance of LEDs devices in visible range does not degrade after fabricating $\mathrm{ZnO}$ nanostructures on top of the devices. The enhanced transmittance of NS- and NR-ZnO is mainly due to the formation of the nanotexturing surface. Rougher surface changed the light trajectory in a chaotic fashion, and the optical phase-space distribution turned into ergodic, resulting in improvement of the light transmittance. These results are in good agreement with previous study on textured ITO/ZnO surface [15]. It 


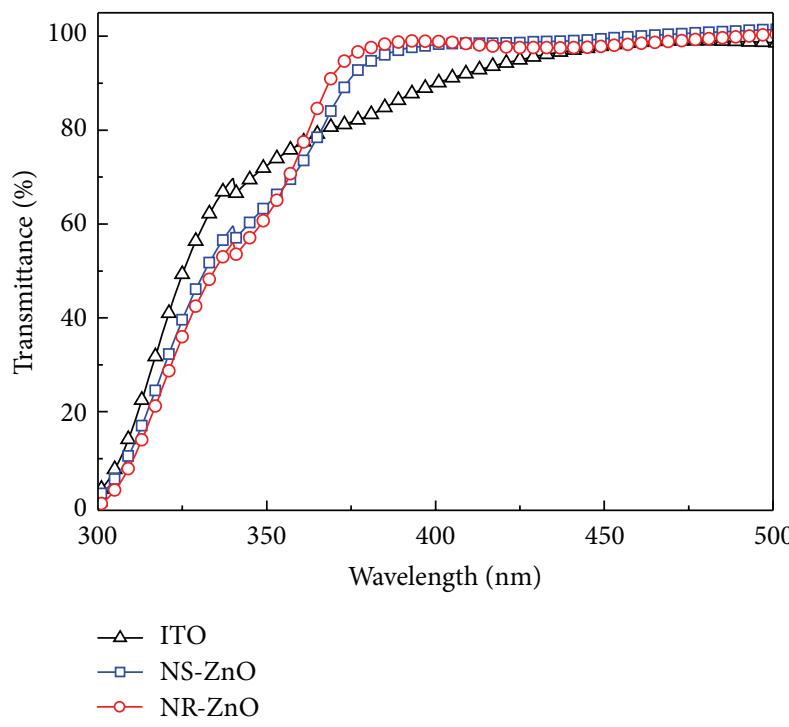

(a)

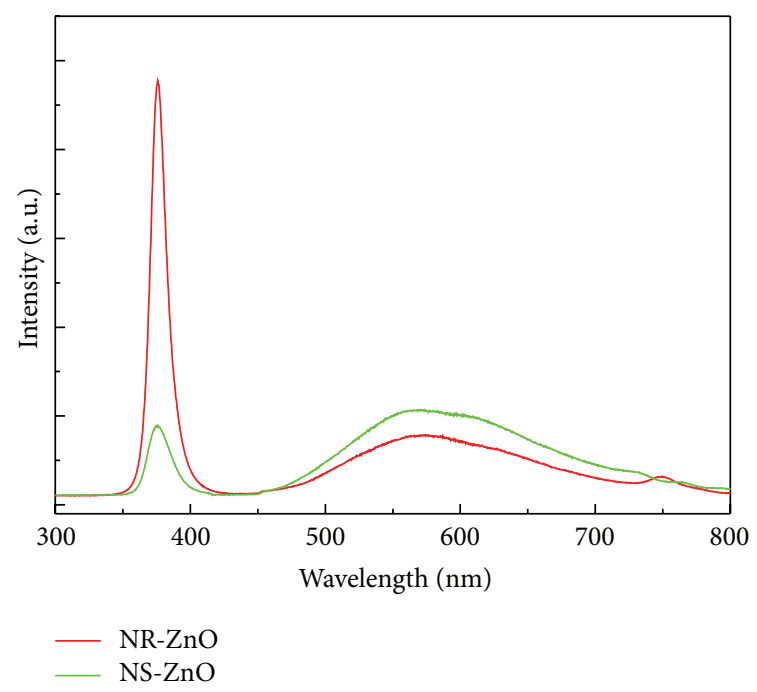

(b)

Figure 3: (a) UV-Vis and (b) PL spectra of NR-ZnO and NS-ZnO.

should be noted that the transmittance of NR- $\mathrm{ZnO}$ is slightly higher than that of NS-ZnO at NUV range $(375 \mathrm{~nm})$. On the other hand, the lower transmittance of NS-ZnO as compared to NR-ZnO has been attributed to the angular randomization of photons inside the nanosheets. Moreover, it is implicitly assumed that natural amorphous characteristic of nanosheets with large amount of defects can further limit the transmission.

Room temperature photoluminescence was measured with $\mathrm{He}-\mathrm{Cd}$ laser excitation at a wavelength of $325 \mathrm{~nm}$ to evaluate the optical properties of $\mathrm{NS}-\mathrm{ZnO}$ versus $\mathrm{NR}-\mathrm{ZnO}$, as shown in Figure 3(b). A main peak around $384 \mathrm{~nm}$ refers to the near band-edge emission. A broad low intensity peak in the visible region is associated with the defect-level emission, which is attributed to deep level defects, such as oxygen vacancies and $\mathrm{Zn}$ interstitial defects. It revealed that NR-ZnO shows better crystalline quality than NS-ZnO because of higher near band-edge emission and lower defectlevel emission peak intensity. This result is an experimental support for our suggestion that the low crystalline quality of nanosheets is responsible for the low optical transmission of nanosheets as compared to nanorods in the UV-Vis spectra.

Electrical characteristics of the C-LED, NS-LED, and NRLED were also studied from the current-voltage $(I-V)$ curves. Figure 4(a) shows that the turn-on voltages of all samples were about $3.28 \mathrm{~V}$ at an injection current of $20 \mathrm{~mA}$ even though the $\mathrm{ZnO}$ nanostructures were grown on ITO layer. No significant difference in the $I-V$ curves was observed at an injection current up to $100 \mathrm{~mA}$. $\mathrm{ZnO}$ nanostructures based LED devices exhibited a slight change in leakage current after fabricating the NR-ZnO and NS-ZnO on top of the devices. The leakage currents were also all around $1.3 \mu \mathrm{A}$ at $-10 \mathrm{~V}$. This small difference in leakage current at the reverse region may originate from the fabrication process of $\mathrm{ZnO}$ nanostructures. However, it would not lead to serious degradation of the electrical properties. Figure 4(b) shows light output power-current $(L-I)$ characteristics of the C-LED, NS-LED, and NR-LED. Compared to C-LED, the optical output power of the NR-LED was dramatically increased with NR-ZnO $(47 \%)$ at an injection current of $100 \mathrm{~mA}$. The enhanced light output power of NR-LED is primarily due to the scattering effect by surface texturing of the NR-ZnO on ITO layer. NR-ZnO structure has a large number of sidewalls and can provide a larger escape cone for the photons in the NR-LED than that in the C-LED. Moreover, the sample with $\mathrm{ZnO}$ nanostructures on ITO layer had the more opportunity to scatter the light at the $\mathrm{ZnO}$ nanostructures, which resulted in an increase in light output power. On the other hand, the light output power of NS-LED was decreased by about $42 \%$. A possible explanation for this observation is that the NR- $\mathrm{ZnO}$ possesses higher crystalline quality than that of NS-ZnO. It is observed that the visible emission of $\mathrm{NS}-\mathrm{ZnO}$ is enhanced and the UV emission is greatly weakened compared to NR$\mathrm{ZnO}$, as shown in Figure 3(b). This result may be due to more defects originating from $\mathrm{Al}$ element doping. Consequently, photons generated from the MQWs of NUV-LEDs can be trapped in defected sites of NS-ZnO, resulting in a significant decrease of the light output power.

Figure 5 shows the micrograph images of the C-LED, NSLED, and NR-LED device operating at an injection current of 20 and $100 \mathrm{~mA}$, respectively. As shown in Figure 5, it is clearly observed that higher intensity of light emission is observed in the top emitting NR-LED due to the formation of a large number of textured surfaces having a larger escape cone. NR-ZnO can also enhance the escape probability. In other words, the photons have multiple opportunities to find the escape cone more easily. However, the light output intensity of NS- $\mathrm{ZnO}$ is decreased because the higher defective NS-ZnO can trap or absorb the photon emitted from the MQWs. We suggest that light extraction efficiency would be 


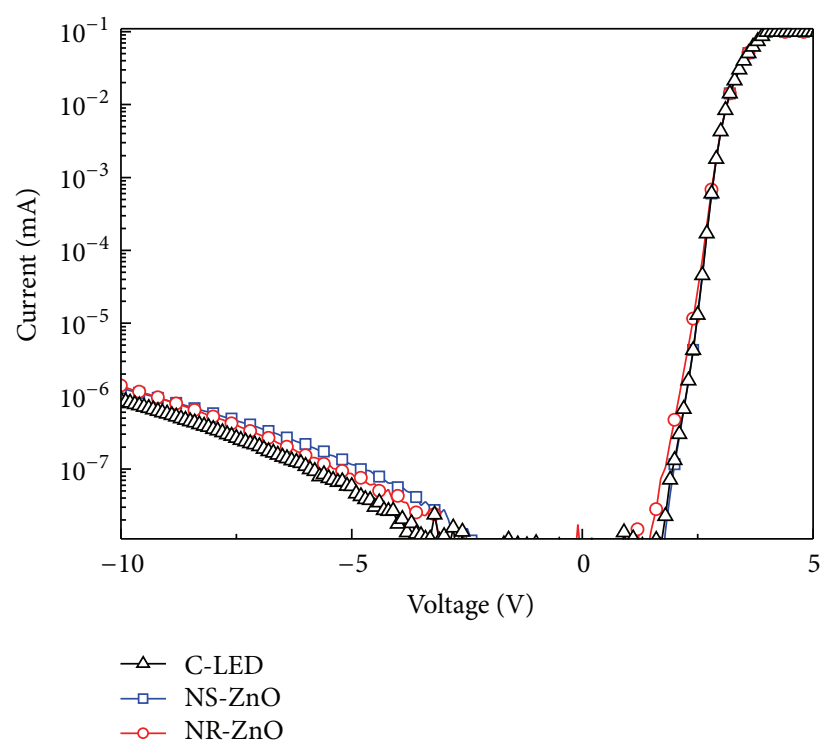

(a)

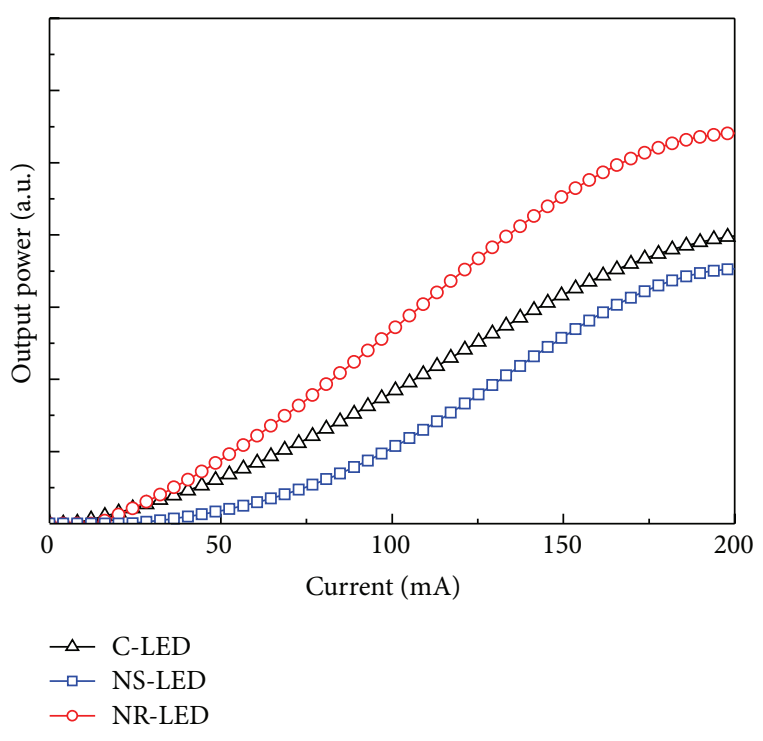

(b)

FIGURE 4: (a) $I-V$ and (b) light output power as a function of applied current of fabricated NR-LED, NS-LED, and C-LED.
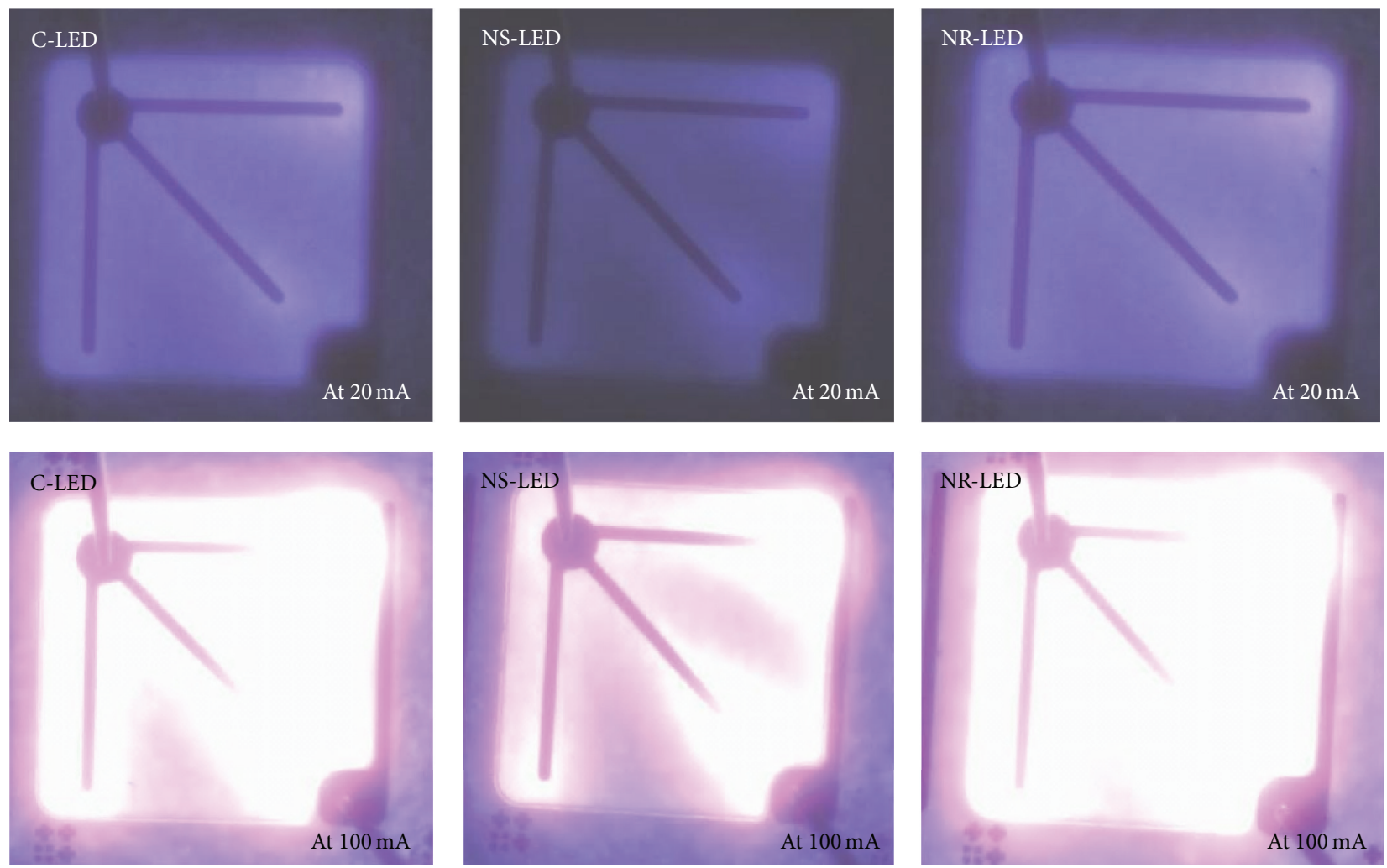

FIGURE 5: Optical micrographs of C-LED, NR-LED, and NS-LED at an operating current of $20 \mathrm{~mA}$ and $100 \mathrm{~mA}$, respectively. 
different depending on the forms of $\mathrm{ZnO}$ in the NUV-LEDs. In summary, $\mathrm{NR}-\mathrm{ZnO}$ structure is more effective on the light extraction for the NUV-LED than NS-ZnO.

\section{Conclusion}

In conclusion, a facile method to enhance light extraction efficiency of $375 \mathrm{~nm}$ NUV-LEDs by $\mathrm{ZnO}$ nanostructures on a planar indium tin oxide (ITO) transparent electrode has been reported. $\mathrm{NR}-\mathrm{ZnO}$ was compared with $\mathrm{NS}-\mathrm{ZnO}$ to elucidate the effect of $\mathrm{ZnO}$ nanostructures on the light output power. At an injection current of $100 \mathrm{~mA}$, the light output power of the NR-LED was enhanced by around $47 \%$ as compared to the C-LED. The increased light extraction by the NR-ZnO is due to the formation of a surface texturing, resulting in an enhancement of the escape probability and the multiple scattering for the photons in the NUV-LED. The light output efficiency of the NS-LED was decreased by $42 \%$ compared to the C-LED due to an increase of the internal reflection and light absorption in the defective NS- $\mathrm{ZnO}$.

\section{Competing Interests}

The authors declare that there is no competing interests regarding the publication of this paper.

\section{Acknowledgments}

This work was supported by Basic Research Program through the National Research Foundation of Korea (NRF) funded by the Ministry of Education (NRF-2014R1A1A2008104 and 2013R1A1A2013044) and ICT \& Future Planning (20100019694) and the BK21 Plus Center for Future Energy Materials and Devices.

\section{References}

[1] D. Birtalan and W. Nunley, Optoelectronics: Infrared-VisibleUltraviolet Devices and Applications, CRC Press, 2nd edition, 2009.

[2] A. Khan, K. Balakrishnan, and T. Katona, "Ultraviolet lightemitting diodes based on group three nitrides," Nature Photonics, vol. 2, no. 2, pp. 77-84, 2008.

[3] J. K. Sheu, S. J. Chang, C. H. Kuo et al., "White-light emission from near UV InGaN-GaN LED chip precoated with blue/green/red phosphors," IEEE Photonics Technology Letters, vol. 15, no. 1, pp. 18-20, 2003.

[4] M. Iwaya, S. Terao, N. Hayashi, T. Kashima, H. Amano, and I. Akasaki, "Realization of crack-free and high-quality thick $\mathrm{Al}_{x} \mathrm{Ga}_{1-x} \mathrm{~N}$ for UV optoelectronics using low-temperature interlayer," Applied Surface Science, vol. 159-160, pp. 405-413, 2000.

[5] Y. A. Xi, K. X. Chen, F. Mont et al., "Comparative study of n-type AlGaN grown on sapphire by using a superlattice layer and a low-temperature AlN interlayer," Journal of Crystal Growth, vol. 299, no. 1, pp. 59-62, 2007.

[6] T. H. Seo, S. J. Chae, B. K. Kim, G. Shin, Y. H. Lee, and E. Suh, "Enhanced light output power of near-ultraviolet lightemitting diodes with au-doped graphene for transparent and current-spreading electrode," Applied Physics Express, vol. 5, no. 11, Article ID 115101, 2012.
[7] J.-P. Shim, T. H. Seo, J. H. Min, C. M. Kang, E.-K. Suh, and D.S. Lee, "Thin Ni film on graphene current spreading layer for GaN-based blue and ultra-violet light-emitting diodes," Applied Physics Letters, vol. 102, no. 15, Article ID 151115, 2013.

[8] D.-S. Han, J.-Y. Kim, S.-I. Na et al., "Improvement of light extraction efficiency of flip-chip light-emitting diode by texturing the bottom side surface of sapphire substrate," IEEE Photonics Technology Letters, vol. 18, no. 13, pp. 1406-1408, 2006.

[9] S. Fan, P. R. Villeneuve, and J. D. Joannopoulos, "Rate-equation analysis of output efficiency and modulation rate of photoniccrystal light-emitting diodes," IEEE Journal of Quantum Electronics, vol. 36, no. 10, pp. 1123-1130, 2000.

[10] T. Fujii, Y. Gao, R. Sharma, E. L. Hu, S. P. DenBaars, and S. Nakamura, "Increase in the extraction efficiency of GaN-based light-emitting diodes via surface roughening," Applied Physics Letters, vol. 84, no. 6, pp. 855-857, 2004.

[11] C. Huh, K.-S. Lee, E.-J. Kang, and S.-J. Park, "Improved light-output and electrical performance of InGaN-based lightemitting diode by microroughening of the $p$-GaN surface," Journal of Applied Physics, vol. 93, no. 11, pp. 9383-9385, 2003.

[12] X. A. Cao, A. P. Zhang, G. T. Dang et al., "Plasma damage in pGaN," Journal of Electronic Materials, vol. 29, no. 3, pp. 256-261, 2000.

[13] T. V. Cuong, H. N. Tien, V. H. Luan et al., "Controlled growth of $\mathrm{ZnO}$ nanomaterials via hydrothermal method: effect of buffer layer," Journal of Nanoscience and Nanotechnology, vol. 12, no. 4, pp. 3313-3316, 2012.

[14] J. P. Cheng, X. B. Zhang, and Z. Q. Luo, "Oriented growth of $\mathrm{ZnO}$ nanostructures on $\mathrm{Si}$ and $\mathrm{Al}$ substrates," Surface and Coatings Technology, vol. 202, no. 19, pp. 4681-4686, 2008.

[15] P. Uthirakumar, J. H. Kang, B. D. Ryu, H. G. Kim, H. K. Kim, and C.-H. Hong, "Nanoscale ITO/ZnO layer-texturing for highefficiency InGaN/GaN light emitting diodes," Materials Science and Engineering: B, vol. 166, no. 3, pp. 230-234, 2010. 

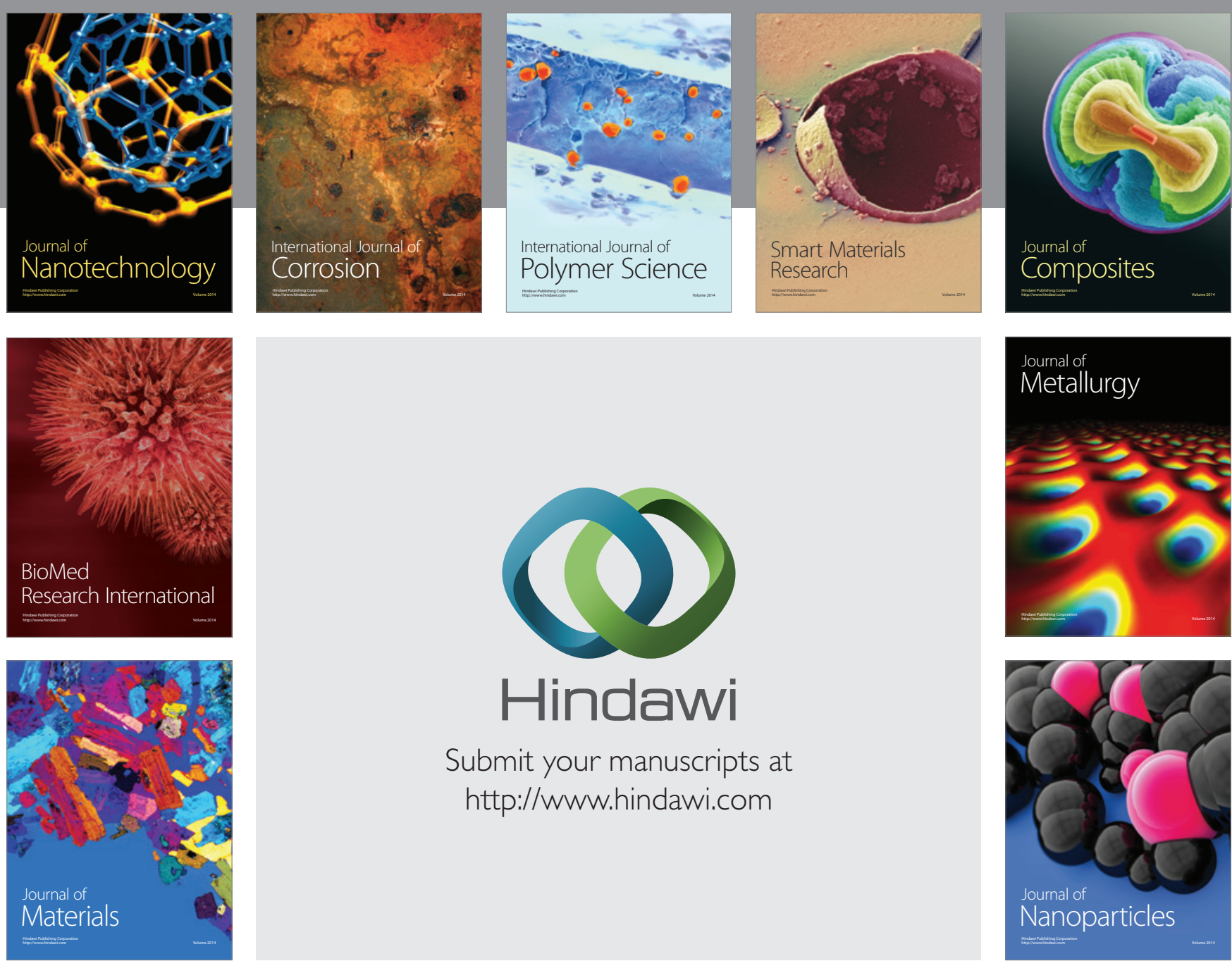

\section{Hindawi}

Submit your manuscripts at

http://www.hindawi.com

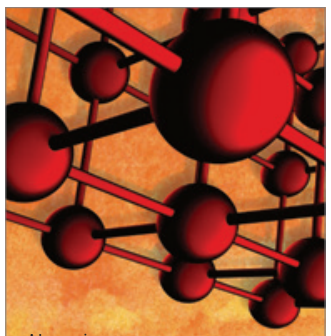

Materials Science and Engineering
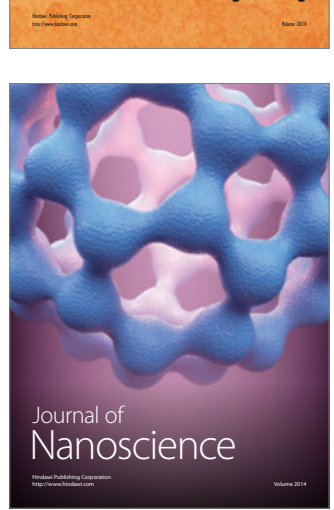
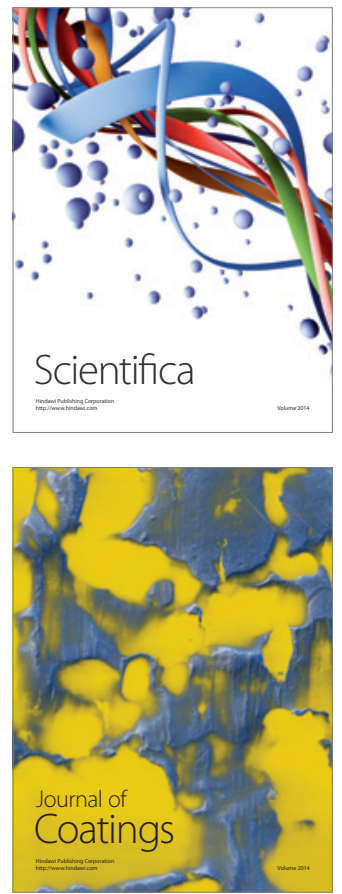
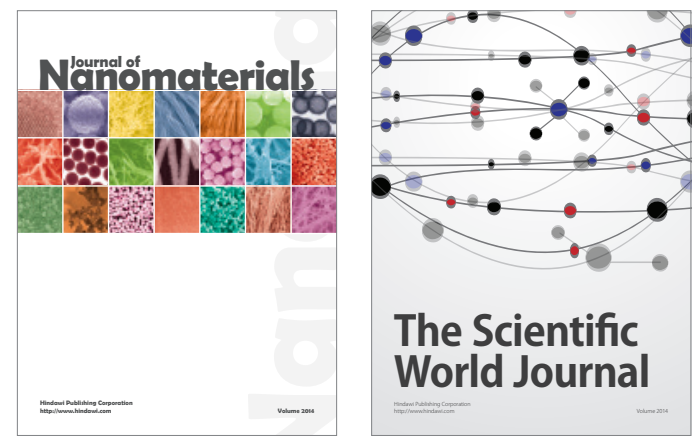

The Scientific World Journal
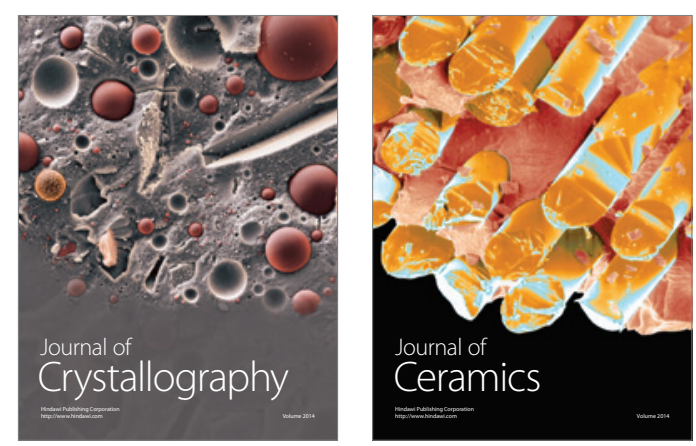
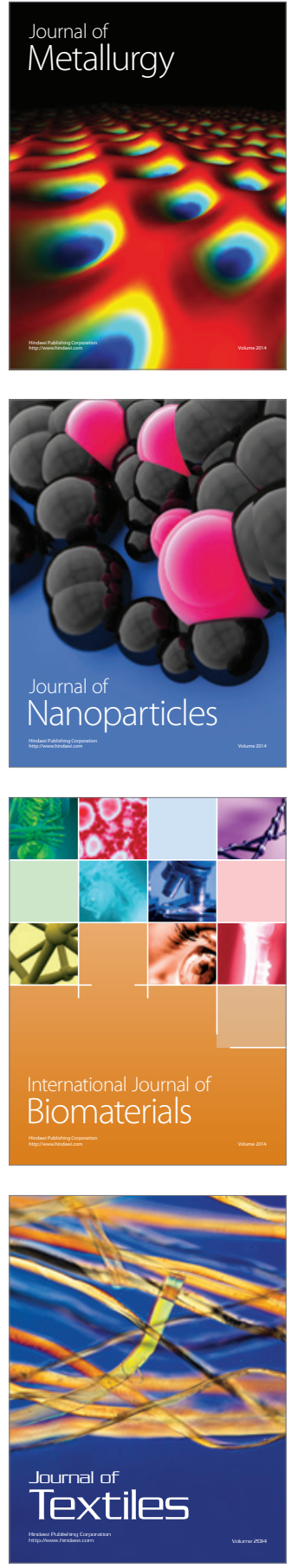